\title{
Structures of light halo nuclei described by an extended framework of antisymmetrized molecular dynamics
}

\author{
N. Furutachi ${ }^{1}$ and M. Kimura ${ }^{2}$ \\ 1 Meme Media Laboratory, Hokkaido University, Sapporo 060-8628, Japan \\ 2 Creative Research Initiative "Sousei”, Hokkaido University, Sapporo 001-0021, Japan
}

\begin{abstract}
We have studied the structures of He isotopes, ${ }^{8} \mathrm{~B}$, and ${ }^{9} \mathrm{C}$ by using an extended framework of antisymmetrized molecular dynamics, which is designed to describe the halo structure and the core excitation simultaneously. The effectiveness of the present framework in describing the halo structure is demonstrated in the calculated results of He isotopes. The structures of ${ }^{8} \mathrm{~B}$ and ${ }^{9} \mathrm{C}$ are discussed focusing on the halo structure and the core excitation in these nuclei. We have found the cluster structures in the cores of these nuclei, which plays an important role in explaining the observed physical quantities.
\end{abstract}

\section{Introduction}

Halo structures have been extensively studied as an interesting subject among exotic structures with unstable nuclei $[1,2]$. In many cases, a halo nucleus is treated as a two- or three-body system consisting of an inert core and one or two loosely bound nucleons. However, in general, unstable nuclei around the neutron drip-line have many valence neutrons, and therefore, the core in the halo nuclei is also an unstable nucleus in many cases. The assumption of a rigid inert core may not be valid in such nuclei. Actually, there have been experimental indications that portray the importance of core excitation. For example, in ${ }^{19} \mathrm{C}$, Glauber model analysis of the interaction cross section and the momentum distribution suggests core excitation of this nucleus [3]. It is difficult to treat such nuclei with few-body models with an assumption of inert core.

Therefore, a theoretical model to investigate the halo structure involving core excitation is necessary. For this purpose, we have developed an extended framework based on antisymmetrized molecular dynamics (AMD) $[4,5]$, the multiple-width Gaussian basis AMD (MAMD). The main improvement in MAMD over the other AMD models is that Gaussian wave packets describing nucleons have different width parameters. The same type of nucleon wave function has also been used in fermionic molecular dynamics (FMD) [6]. However, the present theoretical model has several differences that describe the core excitation more effectively.

To demonstrate the efficiency of the present model in describing the halo structure, we first apply it to He isotopes, where the two-neutron halo structure of ${ }^{6} \mathrm{He}$ is well known. Next, we investigate the structures of ${ }^{8} \mathrm{~B}$ and ${ }^{9} \mathrm{C}$ focusing on their halo structure and core excitation. In ${ }^{8} \mathrm{~B}$, which is well known to have one proton halo structure $[7,8]$, a sizable component of the core-excited ${ }^{7} \mathrm{Be}^{*}\left(J^{\pi}=\right.$ $\left.1 / 2^{-}\right)+p$ configuration has been found by the measure- ment of $\gamma$ rays in coincidence with fragments from the one-proton removal reaction [9]. ${ }^{8} \mathrm{~B}$ is considered to be the lightest nucleus where the halo structure and the core excitation have been known, and therefore, a good starting point to investigate the halo structure involving core excitation. ${ }^{9} \mathrm{C}$ is a candidate for the two-proton halo nucleus. This nucleus has a small two-proton separation energy $(1.43 \mathrm{MeV})$ and a large two-proton removal cross section [10]. It is also interesting to investigate how the halo and core structures of ${ }^{8} \mathrm{~B}$ are changed by adding one more proton.

In the next section, we explain the formulation of MAMD, and Section 3 shows the calculated results for He isotopes, ${ }^{8} \mathrm{~B}$, and ${ }^{9} \mathrm{C}$. The final section summarizes this work.

\section{Theoretical model}

To investigate the halo structure involving core excitation, we have constructed MAMD based on AMD [4,5]. In MAMD, the nucleon wave function $\varphi_{i}(\mathbf{r})$ is described by the superposition of Gaussian wave packets that have different width parameters:

$$
\begin{aligned}
\varphi_{i}(\mathbf{r}) & =\sum_{\alpha}^{N_{i}} C_{i}^{\alpha} \phi_{i}^{\alpha}(\mathbf{r}) \chi_{i}^{\alpha} \tau_{i}, \\
\phi_{i}^{\alpha}(\mathbf{r}) & =\exp \left[-v_{i}^{\alpha}\left(\mathbf{r}-\mathbf{Z}_{i}^{\alpha} / \sqrt{v_{i}^{\alpha}}\right)^{2}\right] .
\end{aligned}
$$

Here, $\chi_{i}^{\alpha}$ is the spin part parameterized by complex number parameter $\xi_{i}^{\alpha}$,

$$
\chi_{i}^{\alpha}=\left(1 / 2+\xi_{i}^{\alpha}\right) \chi_{\uparrow}+\left(1 / 2-\xi_{i}^{\alpha}\right) \chi_{\downarrow},
$$


and $\tau_{i}$ is the isospin component fixed to a proton or a neutron. $\mathbf{Z}_{i}^{\alpha}$ is a complex three-dimensional vector, and $b_{i}^{\alpha}=$ $1 / \sqrt{2 v_{i}^{\alpha}}$ is the width parameter with a real value. The nucleons in the core nucleus with $Z=N$ are expressed by a Gaussian wave packet, whereas the excess $|N-Z|$ nucleons are written by the superposition of two Gaussian wave packets.

We use the effective nuclear force that has central and spin-orbit part. For the central part, Volkov No. 2 force [11] $(\mathrm{m}=0.6$ and $\mathrm{b}=\mathrm{h}=0)$ is used, and the spin-orbit term of G3RS force [12] with the strength parameter $V_{L S}=2000$ $\mathrm{MeV}$ is adopted for the spin-orbit part.

We perform the energy variation using the parity projected wave function $\Phi^{ \pm}=\hat{P}^{ \pm} \Phi_{\text {int }}$, and optimize $\mathbf{Z}_{i}^{\alpha}, \xi_{i}^{\alpha}$, $C_{i}^{\alpha}$ and $v_{i}^{\alpha}$. In this study, the energy variation is performed under the constraint of the proton and neutron root-meansquare (rms) radii $r^{p, n}$ for ${ }^{8} \mathrm{~B}$ and ${ }^{9} \mathrm{C}$, and matter radius $r^{m}$ for He isotopes. The $r^{m, p, n}$ are defined as

$$
r^{m, p, n}=\sqrt{\frac{\left\langle\Phi^{ \pm}\left|\hat{\mathbf{r}}_{m, p, n}^{2}\right| \Phi^{ \pm}\right\rangle}{\left\langle\Phi^{ \pm} \mid \Phi^{ \pm}\right\rangle}} .
$$

Since the extended nucleon wave function prevents the exact separation of the center-of-mass wave function in MAMD, we also constrain $\left\langle\hat{\mathbf{r}}_{g}\right\rangle$ and $\left\langle\hat{\mathbf{p}}_{g}\right\rangle$ to the coordinate origin to fix the center of mass at the origin by adding the constraint potential, as performed by Doté and Horiuchi [13].

After the variation, the optimized wave functions $\Phi^{ \pm}\left(r^{p}, r^{n}\right)$ having various rms radii are projected to an eigenstate of the total angular momentum $J, \Phi_{M K}^{J \pm}\left(r^{p}, r^{n}\right)=$ $\hat{P}_{M K}^{J} \Phi^{ \pm}\left(r^{p}, r^{n}\right)$. Finally, we superpose all the obtained wave functions $\Phi_{M K}^{J_{ \pm}}\left(r^{p}, r^{n}\right)$ by diagonalizing the Hamiltonian. The wave function that describes a certain state is given as

$$
\Psi_{n}^{J \pm}=\sum_{i} c_{i}^{n} \Phi_{M K_{i}}^{J \pm}\left(r_{i}^{p}, r_{i}^{n}\right),
$$

where $c_{i}$ is determined by the Hill-Wheeler equation [14].

We use $\Phi_{\text {int }}, \Phi_{M K}^{J \pm}$, and $\Psi_{n}^{J \pm}$ in the calculation of the physical quantities and analysis. We refer to them as the intrinsic wave function, the angular momentum projected wave function, and GCM wave function, respectively.

\section{Results}

\subsection{He isotopes}

First we apply MAMD to He isotopes, and discuss the effectiveness of the present framework in describing the halo structure.

The binding energies and rms radii of He isotopes are shown in Fig. 1. As clearly seen, binding energies are drastically improved by MAMD, and show a reasonable agreement with the experiments except for ${ }^{10} \mathrm{He}$. The energy difference between MAMD and AMD is largest in ${ }^{8} \mathrm{He}$, where $2.7 \mathrm{MeV}$ is improved by MAMD. In the calculation
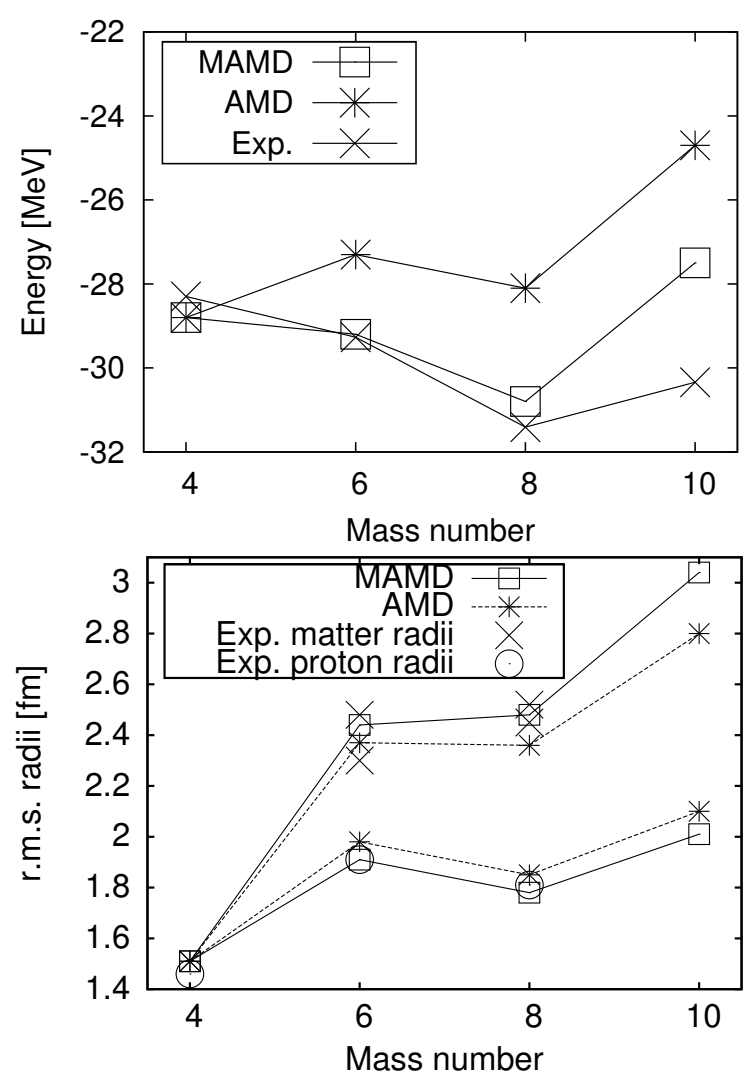

Fig. 1. Energies and radii of He isotopes. The experimental proton radii are taken from Refs. [15-17], and the empirical values of the matter radii are taken from Refs. [18] and [19].

of rms radii, the MAMD results show the neutron radii to be much larger and the proton radii to be smaller compared with the AMD results. The smaller proton radius of 1.91 $\mathrm{fm}$ in ${ }^{6} \mathrm{He}$ completely agrees with the experimental value [15], and that of $1.78 \mathrm{fm}$ in ${ }^{8} \mathrm{He}$ is also closer to the experimental value of $1.81 \mathrm{fm}$ [16].

To discuss how these drastic improvements are achieved in MAMD, we analyze the obtained wave functions of ${ }^{6} \mathrm{He}$ and ${ }^{8} \mathrm{He}$ in detail. First we discuss the results of ${ }^{6} \mathrm{He}$. We analyze the states that have minimum energy among GCM bases, and label those of ${ }^{6} \mathrm{He}$ calculated by MAMD and AMD as state (a) and (b), respectively. Fig. 2 shows the density distributions and the centers of the Gaussian wave packets of state (a) and (b).

The improvement over the AMD result is caused by the simultaneous description of the halo-like spatial extension of the neutron and the shell-like structure. As seen in Fig. 2(a), the centers of the wave packets $\left(\operatorname{Re}\left[\mathbf{Z}_{i}^{\alpha} / \sqrt{v_{i}^{\alpha}}\right]\right)$ in state (a) are approximately localized in two points, which we denote $\mathbf{R}_{c}$ and $\mathbf{R}_{v}$. The halo-like spatial extension of the neutron density distribution in ${ }^{6} \mathrm{He}$ is described by two neutron wave packets at $\mathbf{R}_{v}$, which have considerably large width parameter. The width parameter $b_{i}^{\alpha}=1 / \sqrt{2 v_{i}^{\alpha}}$ of these wave packets is $2.13 \mathrm{fm}$, which is much larger than common width parameter of $1.54 \mathrm{fm}$ in AMD. As clearly seen in Fig. 2(a), the density distribution shows a large spatial extension around $\mathbf{R}_{v}$. Two neutron wave functions at 


\section{$19^{\text {th }}$ International IUPAP Conference on Few-Body Problems in Physics}

$\mathbf{R}_{v}$ approximately couple to the intrinsic spin $S=0$, and describe the di-neutron-like correlation in the outer region of ${ }^{6} \mathrm{He}$. Though state (b) also has two neutron wave packets that are considerably separated from $\alpha$ core, this state fails to describe the spatial extension of the density distribution. Thus, we conclude that the large width parameter is important for describing the spatial extension of the density distribution. At $\mathbf{R}_{c}$, four wave packets forms $\alpha$ particle, and additional two wave packets which are absent in AMD describe the shell-like aspect of ${ }^{6} \mathrm{He}$. If there is only $\alpha$ particle at $\mathbf{R}_{v}$, the spin-orbit force does not work in state (a), because the $\alpha$ and di-neutron are spin-zero systems. However, expectation value of the spin-orbit force of state (a) is $-2.5 \mathrm{MeV}$, whereas that of state (b) is approximately zero. In state (a), a $p 3 / 2$ configuration component described by two neutron wave functions at $\mathbf{R}_{v}$ brings about a considerable expectation value of the spin-orbit force that is important for the binding of ${ }^{6} \mathrm{He}$.
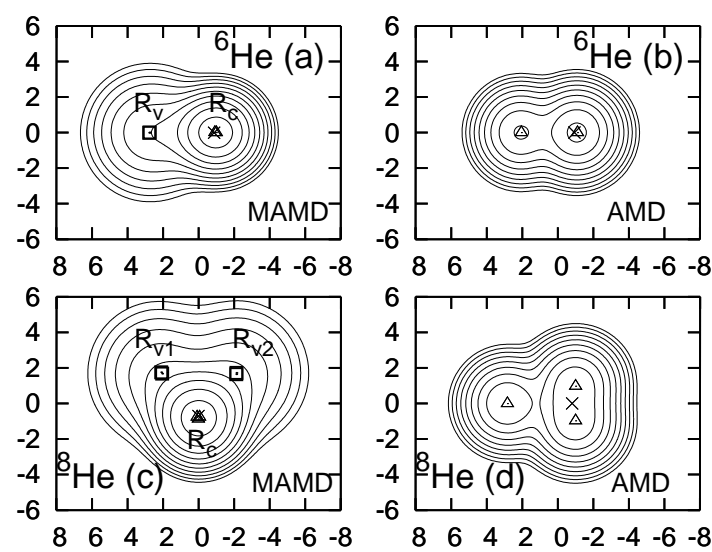

Fig. 2. Density distributions of ${ }^{6} \mathrm{He}$ and ${ }^{8} \mathrm{He}$ calculated with the intrinsic wave functions. Units on both axes are fm. Contour lines are plotted in logarithmic scale. Centers of Gaussian wave packets are plotted by symbols. Crosses represent the proton wave packets, whereas triangles and squares represent the neutron wave packets that are narrow and wide, respectively. $\mathbf{R}_{c}$ and $\mathbf{R}_{v}$ in (a) and (c) denote the positions of localized wave packets.

Next, we discuss the results of ${ }^{8} \mathrm{He}$. As in the case of ${ }^{6} \mathrm{He}$, we analyze the states that have minimum energy among the GCM bases. We label the states calculated by MAMD and AMD as states (c) and (d), respectively. Figure 2 shows the density distributions and the centers of Gaussian wave packets of states (c) and (d).

We found similar improvements with the case of ${ }^{6} \mathrm{He}$ in ${ }^{8} \mathrm{He}$. In state (c), the centers of the wave packets are localized at three points, which we denote $\mathbf{R}_{v 1}, \mathbf{R}_{v 2}$, and $\mathbf{R}_{c}$. Two neutron wave packets at $\mathbf{R}_{v 1}$ and $\mathbf{R}_{v 2}$, which have large width parameter, describe the spatial extension of the neutrons. Width parameter of these wave packets is 2.50 $\mathrm{fm}$, which is considerably larger than common width parameter $1.54 \mathrm{fm}$ of state $(\mathrm{d})$. The density distribution of state (c) clearly shows a spatial extension around $\mathbf{R}_{v 1}$ and $\mathbf{R}_{v 2}$. As in the case of ${ }^{6} \mathrm{He}$, wide wave packets are essential in describing the spatial extension of the density distribution. Four neutron wave packets at $\mathbf{R}_{c}$, where other four wave packets form $\alpha$ particle, describe the shell-like aspect of ${ }^{8} \mathrm{He}$. Although two neutron wave functions at $\mathbf{R}_{v 1}$ and $\mathbf{R}_{v 2}$ roughly couple to intrinsic spin $S=0$, the expectation value of the spin-orbit force of state (c) is $-9.7 \mathrm{MeV}$, and largely contribute to the total binding energy of ${ }^{8} \mathrm{He}$, whereas that of state (d) is $-2.9 \mathrm{MeV}$. The shell-like component with a $p 3 / 2$ configuration described by four neutron wave packets at $\mathbf{R}_{c}$ brings about this result.

Consequently, the simultaneous description of the halolike spatial extension of the neutron and the shell-like structure brings about the large difference between the descriptions in MAMD and AMD, which results in the reasonable agreement with the experimental results of the binding energy and radii in ${ }^{6} \mathrm{He}$ and ${ }^{8} \mathrm{He}$.

\section{$3.2{ }^{8} \mathrm{~B}$ and ${ }^{9} \mathrm{C}$}

We applied MAMD to ${ }^{8} \mathrm{~B}$ and ${ }^{9} \mathrm{C}$ to investigate the halo structure and the core excitation of these nuclei. Twodimensional GCM calculations were performed using the constraints on neutron and proton radii $\left(r^{n}, r^{p}\right) . r^{n}$ is chosen in the range from 1.9 to $2.7 \mathrm{fm}$ with an interval of 0.2 $\mathrm{fm}$, and $r^{p}$ is chosen from $r^{n}+0.1 \mathrm{fm} r^{n}+1.0 \mathrm{fm}$ with an interval of $0.1 \mathrm{fm}$. Consequently, 50 bases are incorporated into the GCM calculation of ${ }^{8} \mathrm{~B}$ and ${ }^{9} \mathrm{C}$.

Table 1 shows the binding energies, radii, electric quadrupole moment, and magnetic dipole moment of ${ }^{8} \mathrm{~B}$ and ${ }^{9} \mathrm{C}$. The binding energies and radii of ${ }^{8} \mathrm{~B}$ and ${ }^{9} \mathrm{C}$ show a reasonable agreement with the experimental values, although the binding energy of ${ }^{8} \mathrm{~B}$ is slightly underestimated. As clearly seen, almost all of the physical quantities are improved by MAMD, especially the improvement in the binding energy of ${ }^{9} \mathrm{C}$ is significant. Although the electric quadrupole moment of ${ }^{8} \mathrm{~B}$ and magnetic dipole moment of ${ }^{9} \mathrm{C}$ show slight disagreement with the experimental values, MAMD describes the core excitation that is essential to reproduce these quantities, as will be discussed below.

MAMD result shows that ${ }^{8} \mathrm{~B}$ has the weakly bound $\alpha+{ }^{3} \mathrm{He}+p$ three-body system. Figure 3 shows the twodimensional energy surface of ${ }^{8} \mathrm{~B}$. The energy surface shows a noticeable flatness over a wide area. We discuss the structure of ${ }^{8} \mathrm{~B}$ by analyzing the structure change on the energy surface. For this purpose, we invoke three states from the energy surface. One is the energy minimum state that we label state (a), and others are state (b) and (c) that have a larger proton and a neutron radii, respectively. In Fig. 3, positions on the energy surface and the density distributions of these states are shown. The density distribution and the localization of the wave packets in states (a), (b), and (c) indicate the development of the $\alpha+{ }^{3} \mathrm{He}$ cluster structure in the ${ }^{7} \mathrm{Be}$ core. The distance between the core and one proton develops as $r^{p}$ increases, as represented by state (b), where the development of one proton halo is clearly seen. On the other hand, the relative distance between the $\alpha$ and ${ }^{3} \mathrm{He}$ clusters increases as $r^{n}$ increases, as represented by state (c). By considering these structure change on the energy surface and the noticeable flatness of 
EPJ Web of Conferences

Table 1. Energy, radii, magnetic dipole and electric quadrupole moments of ${ }^{8} \mathrm{~B}$ and ${ }^{9} \mathrm{C}$. The empirical values of the radii are taken from Ref. [20] for ${ }^{8} \mathrm{~B}$, and Ref. [21] for ${ }^{9} \mathrm{C}$. The Schmidt value of the magnetic dipole moment of ${ }^{9} \mathrm{C}$ is shown in parentheses.

\begin{tabular}{cccccccc}
\hline & & B.E $[\mathrm{MeV}]$ & $\sqrt{\left\langle\hat{\mathbf{r}}_{m}^{2}\right\rangle}[\mathrm{fm}]$ & $\sqrt{\left\langle\hat{\mathbf{r}}_{p}^{2}\right\rangle}[\mathrm{fm}]$ & $\sqrt{\left\langle\hat{\mathbf{r}}_{n}^{2}\right\rangle}[\mathrm{fm}]$ & $\mu\left[\mu_{N}\right]$ & $Q\left[e \mathrm{fm}^{2}\right]$ \\
\hline${ }^{8} \mathrm{~B}\left(2^{+}\right)$ & MAMD & 35.7 & 2.50 & 2.64 & 2.24 & 0.95 & 4.3 \\
& AMD & 34.7 & 2.48 & 2.60 & 2.26 & 0.96 & 4.2 \\
& Exp. & 37.379 & 2.55 & 2.76 & 2.16 & 1.04 & 6.45 \\
${ }^{9} \mathrm{C}\left(3 / 2^{-}\right)$ & MAMD & 39.2 & 2.40 & 2.54 & 2.08 & -1.73 & -3.2 \\
& AMD & 36.1 & 2.26 & 2.35 & 2.07 & -1.72 & -2.7 \\
& Exp. & 39.716 & 2.42 & & & -1.39 (Sch. -1.91$)$ & \\
\hline
\end{tabular}
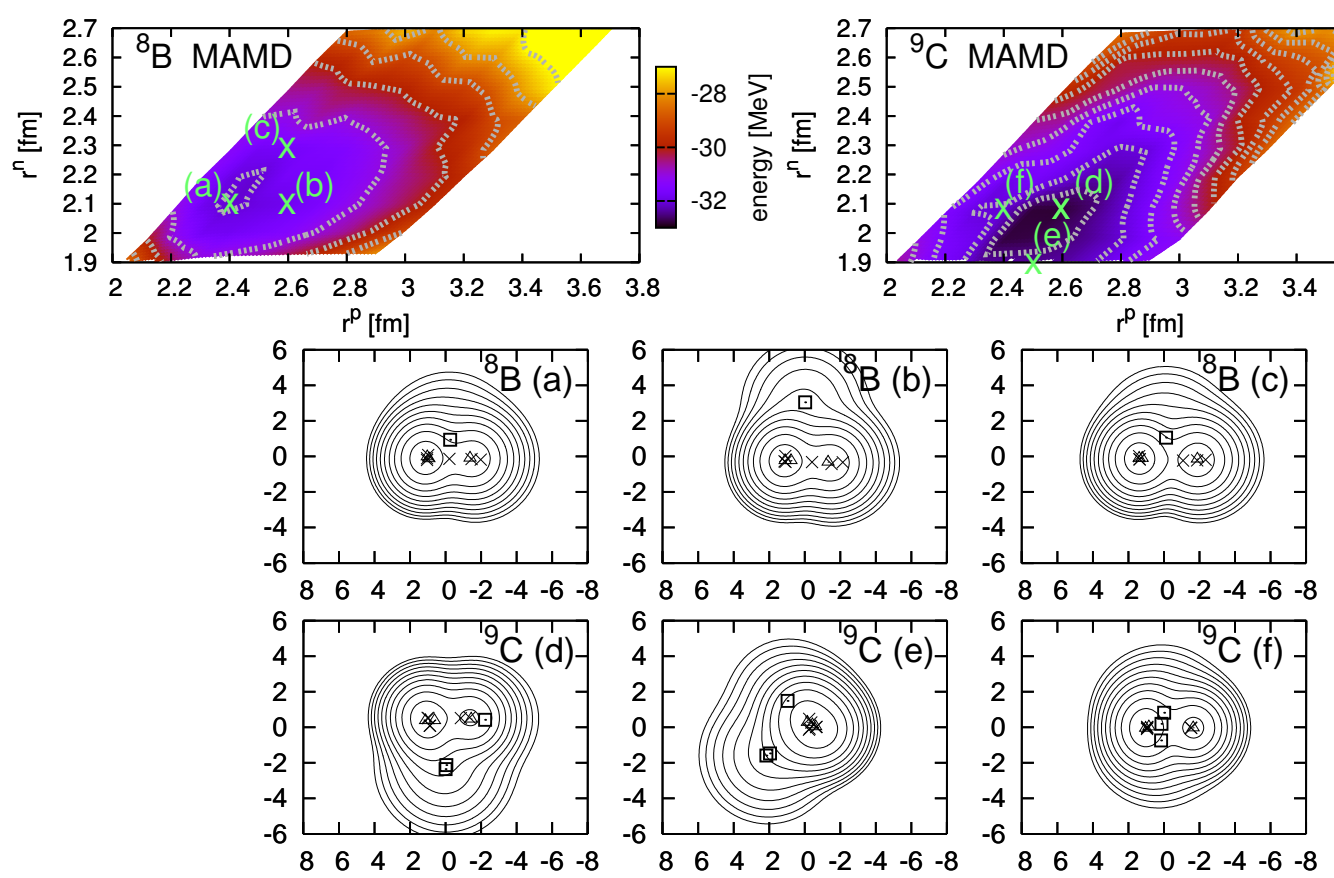

Fig. 3. Energy surface and the density distribution of ${ }^{8} \mathrm{~B}$ and ${ }^{9} \mathrm{C}$. Energy surface is calculated with the angular momentum projected wave functions $\left(2^{+}\right.$for ${ }^{8} \mathrm{~B}$ and $3 / 2^{-}$for $\left.{ }^{9} \mathrm{C}\right)$, and contour lines are plotted with intervals of $1 \mathrm{MeV}$. Centers of Gaussian wave packets are plotted by symbols in the density distribution. Triangles represent the neutron wave packets, whereas crosses and squares represent the proton wave packets that are narrow and wide, respectively.

the energy surface, we conclude that ${ }^{8} \mathrm{~B}$ is a weakly bound $\alpha+{ }^{3} \mathrm{He}+p$ system.

Description of the weakly bound three-body system of ${ }^{8} \mathrm{~B}$ is important for understanding the properties of ${ }^{8} \mathrm{~B}$. The GCM uses $r^{p}$ and $r^{n}$ as generator coordinates, which improves the description of the one-proton halo and the cluster structure in the core, increase the binding energy by 3.6 MeV above that of state (a). The proton and neutron radii are also considerably changed from those of state (a) by GCM. The development of the cluster structure in the core is important for describing the large neutron radius, whereas the development of the one-proton halo is important for describing the large proton radius.

It has been proposed that the observed large quadrupole moment $\left(6.45 e \mathrm{fm}^{2}\right.$ [22]) is an indication of the development of the proton halo structure [23]. However, by analyzing the states on the energy surface, we found that the development of the proton halo structure does not contribute to the increase of the quadrupole mo- ment. Instead, the quadrupole moment is sensitive to the relative distance between the $\alpha$ and ${ }^{3} \mathrm{He}$ clusters. In fact, the calculated quadrupole moment increases by GCM as a result of the mixing of the state with the developed cluster structure in the core. Therefore, the observed large quadrupole moment is considered to be an indication of the development of the cluster structure. Although the quantitative reproduction is not sufficient in this calculation, it is important that the cluster structure in the core that is essential for the reproduction of the quadrupole moment is described in MAMD together with the halo structure.

In ${ }^{9} \mathrm{C}$, both the cluster structure and the shell-like structure in the core are important. Figure 3 shows the twodimensional energy surface of ${ }^{9} \mathrm{C}$. Energy surface shows a valley around the $r^{p}=r^{n}+0.5 \mathrm{fm}$ line, which means the development of the large neutron skin thickness in this system. We have found three characteristic structures in ${ }^{9} \mathrm{C}$. One structure is represented by the energy minimum state which we label state (d), and other structures are found 
$19^{\text {th }}$ International IUPAP Conference on Few-Body Problems in Physics

in state (e) and (f) that have smaller neutron and proton radii, respectively. The density distribution and the localization of the wave packets of state (d) indicate the development of the $\alpha+{ }^{3} \mathrm{He}$ cluster structure in the core. This structure appears in a wide region of the energy surface. The relative distance between the $\alpha$ and ${ }^{3} \mathrm{He}$ clusters in the core increases as a function of $r^{n}$, whereas the relative distance between the core and the two protons increases as a function of $r^{p}$. On the other hand, state (e) has a structure that is clearly different from state (d). In this state, the core has a shell-like one-center structure. This structure appears where the $r^{n}$ decreases from state (d). In state (f), the core nucleons form a two-center core, as do those of state (d). However, unlike state (d), state (f) shows that two protons are not separated from the core. This structure appears where $r^{p}$ decreases from state $(\mathrm{d})$.

Both states that have a cluster structure and a shell-like structure in the core contribute greatly to the properties of ${ }^{9} \mathrm{C}$. For the total binding of ${ }^{9} \mathrm{C}$, the energy minimum state (d), which has a moderately developed cluster structure in the core, is insufficient, but the superposition of the states that have a shell-like structure and a pronounced cluster structure in the core is necessary. The binding energy calculated with the GCM wave function is larger than that of state (d) by $3.6 \mathrm{MeV}$.

The cluster structure in the core is important in understanding the observed magnetic moment, which shows a large deviation from the Schmidt value. If the core has a shell-like structure, the magnetic moment is close to the Schmidt value, as represented by state (e) that has the magnetic moment of $-1.94\left[\mu_{N}\right]$. However, if the core has a cluster structure, the absolute value of the magnetic moment decreases because of the increase of the orbital magnetic quantum number of protons. Actually, the magnetic moment of state (d) that has the cluster structure in the core is $-1.43\left[\mu_{N}\right]$, which is largely deviated from the Schmidt value. In the magnetic moment calculated with the GCM wave function, deviation from the Schmidt value originate from the superposition of the states which have the cluster structure in the core.

\section{Summary}

We have applied MAMD to He isotopes, ${ }^{8} \mathrm{~B}$ and ${ }^{9} \mathrm{C}$. MAMD is an extended framework of AMD to describe the halo structure and the core excitation simultaneously. Effectiveness of MAMD in describing the halo structure was discussed thorough the application to He isotopes, and the structures of ${ }^{8} \mathrm{~B}$ and ${ }^{9} \mathrm{C}$ were investigated focusing on the halo structure and the core excitation in these nuclei.

In He isotopes, We have shown that the calculated binding energies and radii are drastically improved over the results of AMD, and reasonably agree with the experimental values. The improvement was a result of the simultaneous description of the halo-like spatial extension of the neutron density distribution and the shell-like structure.

In ${ }^{8} \mathrm{~B}$ and ${ }^{9} \mathrm{C}$, we found the cluster structure in the core of these nuclei with the halo-like spatial extension of the proton density distribution. We have found that ${ }^{8} \mathrm{~B}$ is a weakly bound $\alpha+{ }^{3} \mathrm{He}+p$ system, and the development of the cluster structure in the core is essential to explain the observed large electric quadrupole moment. In ${ }^{9} \mathrm{C}$, both the cluster structure and the shell-like structure in the core are important. To explain the observed magnetic dipole moment, increase of the orbital magnetic quantum number of protons that originate from the development of the cluster structure in the core is important. Adding to that, for the binding energy of this nucleus, the superposition of the states that have the shell-like structure and the cluster structure in the core is important.

The effectiveness of MAMD in describing the halo structure was confirmed in the result of He isotopes, and we found the core excitation with the halo-like spatial extension of the proton density distribution in ${ }^{8} \mathrm{~B}$ and ${ }^{9} \mathrm{C}$ by applying MAMD. We intend to apply this framework to the investigation of the heavier halo nuclei that is expected to have core excitation.

\section{Acknowledgements}

The numerical calculations were performed on the Altix3700 BX2 at YITP in Kyoto University.

\section{References}

1. I. Tanihata, J. Phys. G: Nucl. Part. Phys. 22 (1996), 157.

2. B. Jonson, Phys. Rep. 389 (2004), 1.

3. R. Kanungo, I. Tanihata, Y. Ogawa, H. Toki and A. Ozawa, Nucl. Phys. A 677 (2000), 171.

4. Y. Kanada-En'yo, H. Horiuchi and A. Ono, Phys. Rev. C 52 (1995), 628.

5. Y. Kanada-En'yo, M. Kimura and H. Horiuchi, C. R. Phys. 4 (2003), 497.

6. T. Neff, H. Feldmeier and R. Roth, Nucl. Phys. A 752 (2005), 321c.

7. W. Schwab et al., Z. Phys. A 350 (1995), 283.

8. M. H. Smedberg et al., Phys. Lett. B 452 (1999), 1.

9. D. Cortina-Gil et al., Nucl. Phys. A 720 (2003), 3.

10. R. E. Warner et al., Phys. Rev. C 69 (2004), 024612.

11. A. B. Volkov, Nucl. Phys. 74 (1965), 33.

12. N. Yamaguchi, T. Kasahara, S. Nagata and Y. Akaishi, Prog. Theor. Phys. 62 (1979), 1018.

13. A. Doté and H. Horiuchi, Prog. Theor. Phys. 103 (2000), 261.

14. D. L. Hill and J. A. Wheeler, Phys. Rev. 89 (1953), 1102.

15. L. -B. Wang et al., Phys. Rev. Lett. 93 (2004), 142501.

16. P. Mueller et al., Phys. Rev. Lett. 99 (2007), 252501.

17. E. Borie and G. A. Rinker, Phys. Rev. A 18 (1978), 324.

18. I. Tanihata et al., Phys. Lett. B 206 (1988), 592.

19. G. D. Alkhazov et al., Phys. Rev. Lett. 78 (1997), 2313.

20. F. Negoita et al., Phys. Rev. C 54 (1996), 1787.

21. A. Ozawa et al., Nucl. Phys. A 608 (1996), 63.

22. T. Sumikama et al., Phys. Rev. C 74 (2006), 024327.

23. T. Minamisono et al., Phys. Rev. Lett. 69 (1992), 2058. 